\title{
Modeling and Analysis of American Chestnut Populations Subject to Various Stages of Infection
}

\author{
Anita Davelos Baines ${ }^{1, *}$, Eric Alan Eager ${ }^{2}$, Andrew M. Jarosz ${ }^{3}$
}

\begin{abstract}
American chestnuts, Castanea dentata, were once a dominant tree in eastern deciduous forests of the United States before the chestnut blight fungus, Cryphonectria parasitica, was introduced unintentionally in the early 1900s in New York. This fungus rapidly devastated American chestnut populations until a hypovirus infection of the fungus began to reduce pathogen virulence on chestnut trees. The subsequent reappearance of large reproducing chestnut trees, associated with a large proportion of blight-infected isolates being parasitized by this hypovirus, is currently taken to indicate recovery of American chestnut populations. We explore, using previously-established matrix population models, the dynamics of healthy, fungus-infected, and hypovirus-infected American chestnut populations to test the efficacy of this recovery. Our main result is that populations transitioning from being fungus-infected to hypovirus-infected are predicted to show large transient amplifications as a result of demographic transitions, only to decline asymptotically to zero, and this result is robust to uncertainty in fecundity values. Our results suggest that the current recovery of the American chestnut population may be a transient phenomenon and that more conservation efforts may be necessary to ensure its long-term persistence.
\end{abstract}

Keywords: basic reproduction number, Cryphonectria parasitica, Castanea dentata, fungus infection, matrix population models

\section{Introduction}

American chestnuts, Castanea dentata (Marsh.) Borkh., were once a dominant tree in eastern deciduous forests of the United States with an estimated 4 billion individual trees [24]. The chestnut blight fungus, Cryphonectria parasitica (Murrill) Barr, was introduced unintentionally in the early 1900s in New York [21], and it rapidly devastated populations of the American chestnut. Branches and trunks infected with the fungus are girdled by cankers and die. The root systems are not infected, thus they may produce new uninfected sprouts from the root collar. These new sprouts eventually become infected, thus perpetuating the epidemic. Double-stranded RNA (dsRNA) hypovirus infection of the fungus reduces pathogen virulence (rate of canker expansion) on chestnut trees [19]. Trees infected with a hypovirus-containing isolate of the pathogen may produce wound callus tissue, which reduces canker expansion rates and prevents the fungus from girdling the branch or trunk. Trees producing these non-lethal (non-girdling) cankers are said to be recovering. Branches

\footnotetext{
${ }^{1}$ Department of Biology, University of Wisconsin-La Crosse, La Crosse, WI

${ }^{2}$ Mathematics Department, University of Wisconsin-La Crosse, La Crosse, WI

${ }^{3}$ Departments of Plant Biology and Plant, Soil \& Microbial Sciences, Michigan State University, East Lansing, MI

${ }^{*}$ Correspondence: abaines@uwlax.edu
} 
and trunks with non-girdling cankers often have increased longevity compared to those with lethal cankers [7].

Recovery of European chestnuts, C. sativa, from blight is widespread in Europe and is associated with the presence of hypoviruses [16]. Attempts to introduce hypoviruses as biological control agents of $C$. parasitica in the eastern United States have largely failed to stop the spread of the disease (reviewed in [19]). In contrast, there has been natural spread of hypoviruses and some recovery of individual trees within naturalized American chestnut populations in Michigan [12]. A study to examine the population-level effects of hypovirus infection of C. parasitica on American chestnut populations using population projection matrices [2] suggested that treatment of diseased American chestnut populations with hypovirus could result in the long-term persistence of American chestnut populations [6]. Three different matrix models were used to compare asymptotic population growth rate $\lambda$ and stable stage (size) distributions of healthy, diseased (blight-infected), and recovered (hypovirus-infected) American chestnut populations in Michigan. Population growth rates did not appear to differ significantly from one for all population types, but stable stage distributions of healthy and hypovirus-infected populations were more similar to each other than to blight-infected populations, suggesting that, at least demographically, a legitimate population-level recovery was occurring at these sites.

While population dynamics is a relatively old area of theoretical ecology, since the publication of the study in Davelos and Jarosz [6] much work has been done to more thoroughly understand structured population dynamics. One important line of work has been the appreciation for, and the development of, measurements of transient dynamics $[14,17,18,3,29,30,11,26,27,9,28,15]$. A population's transient dynamics - the fluctuation and oscillation in both population growth rate and population size over (relatively) short time intervals caused by populations in unstable stage distributions - can vary substantially from a population's asymptotic dynamics [20, 1]. Additionally, the sensitivity of transient dynamics to changes in model parameters can be profoundly different than the sensitivity of $\lambda$ to changes in these same model parameters [28]. Thus, population ecologists need to study both asymptotic population measurements of population growth and measurements of transient dynamics to have the most well-informed approach to management and conservation of populations of interest $[11,1]$.

In this paper we use the basic reproduction number as defined in Cushing [4] to show that the differences in population viability between healthy, diseased and recovered populations are actually not trivial, and that the hypovirus-infected populations modeled in Davelos and Jarosz [6], despite having growth rates within 2 percent of one, are actually quite far away from being able to persist long-term. This poses an interesting ecological question: How, then, does one explain the observed recovery of hypovirus-infected chestnut populations discussed in Davelos and Jarosz [6]? To answer this question, we use population inertia $p_{\infty}[18,28]$ to show that the observed recovery of $C$. dentata populations in Michigan may in fact be the product of transient dynamics alone. We show that population inertia when transitioning from a healthy population to a fungus-infected population is well below one, signalling a transient attenuation. This calculation, coupled with a significant decrease in the basic reproduction number suggests, as expected, that chestnut blight has a significant negative impact on $C$. dentata at the population level. However, when transitioning from a blight-infected population to a hypovirus-infected one the results are mixed: Population inertia is much larger than one, signalling transient amplification, while the basic reproduction number for the hypovirus-infected population is smaller than that for the fungus-infected population, predicting an eventual population decline. We show that this finding is robust to reasonable uncertainty in fecundity estimates, which are the most uncertain estimates in the study by Davelos and Jarosz [6] (discussed below), and propose future avenues of research finding conservation measures that will more effectively increase the chances of long-term population persistence for $C$. dentata. 


\section{Methods and Models}

\subsection{Basic theory and prior results}

In this paper we work with density-independent population projection matrix models

$$
n_{t+1}=A n_{t}
$$

as seen in Caswell [2]. Here, $n_{t}$ is a vector such that the $i$ th element of $n_{t}$ is the total population of $C$. dentata in stage $i$ at time $t$. $A$ is the population projection matrix, where the $i, j$ th element of $A$ is the expected number of individuals in stage $i$ produced by an individual in stage $j$ in each time-step. The basic theory of population projection matrix models is well developed, and can be summarized as follows [4]: if the matrix $A$ is primitive (i.e., there is a positive probability that each stage can eventually contribute to every other stage), then there exists a constant $c$ such that

$$
n_{t} \approx c \lambda^{t} w
$$

as $t \rightarrow \infty$, independent of initial population $n_{0}$, where $\lambda$ is the largest eigenvalue of $A$ (which is real, positive, and unique) and $w$ is the eigenvector associated with $\lambda$ (which is real, positive, and unique). The value $\lambda$ is referred to as the asymptotic growth rate (or finite rate of population increase in [6]) and $w$ the stable stage distribution. If one normalizes $w$ so that its entries sum to one, it yields the proportion of individuals in each of the population's stages as $t \rightarrow \infty$.

We use the matrices developed in Davelos and Jarosz [6], where elements of the transition matrices used in the population projection models were based on data collected annually in the Missaukee, Leelanau, County Line, Frankfort, and Stivers sites in the northern lower peninsula of Michigan, due to their similar population sizes and site characteristics (see [6] for a more thorough description of the population sites). Three population projection matrices were created, one for the two healthy populations (Leelanau and Missaukee Healthy), one for the two populations still experiencing significant fungus-induced epidemics (Missaukee Diseased and Stivers) and one for the two populations that have experienced perceived recovery due to hypovirus infection (Frankfort and County Line). Each model assumed eight population stages, and thus $n_{t}$ from (1) is an 8-dimensional vector and $A$ is a 8 by 8 matrix. All individuals within a population that were greater than $100 \mathrm{~cm}$ in height (assigned stages 5-8 in the model) were measured each year; therefore confidence in these transition probabilities is very high. To estimate transition probabilities for smaller individuals (stages 1-4 in the model), permanent plots were established and all small individuals (less than $100 \mathrm{~cm}$ in height) were measured because it was not feasible to measure every small individual within each population. True seedlings and other small individuals were assigned to different stage classes (stage 1 for true first year seedlings, stages 2-4 for other small individuals) due to differences in survival probability [5]. Numbers of individuals in these plots were scaled to account for the difference in the area of the plots versus the area of the entire population. The placement of the plots was not truly random and this scaling was not exact for the populations so there is likely to be some uncertainty in these probabilities. To estimate reproduction for each tree, the number of branches with burrs was counted for each tree. Three of these branches were selected haphazardly and the number of burrs on each was counted. These estimates have the greatest uncertainty as it was often difficult to view the entire crown of a tree (even with binoculars) to accurately count the number of branches with burrs and the number of burrs. These values were used to estimate the average number of seedlings per individual in each stage and were the measure of fecundity used in the matrix projections. Due to the high uncertainty in fecundity values relative to those of the parameters modeling stage transitions, we will only explore population dynamics subject to uncertainty in fecundity values, and leave uncertainty with regard to stage transitions to the Discussion. 
We will refer to the matrices for the healthy, diseased (fungus-infected) and recovered (hypovirus-infected) populations as $A_{H}, A_{D}$ and $A_{R}$, respectively (parameter values for these matrices can be found in Table 3 in [6]). Each of these matrices is primitive, meaning that the leading eigenvalues, which are $\lambda_{H}=1.014, \lambda_{D}=0.995$, and $\lambda_{R}=0.987$, are the asymptotic growth rates of the populations in each disease scenario. Notice that these $\lambda$ values are slightly different than those reported in [6], who used an average of the growth rates in Table 2 of that paper to compute a rough estimate of the growth in each scenario. The leading eigenvectors, which are

$$
\begin{aligned}
w_{H} & =\left[\begin{array}{llllllll}
0.16 & 0.42 & 0.06 & 0.16 & 0.09 & 0.07 & 0.01 & 0.02
\end{array}\right]^{T}, \\
w_{D} & =\left[\begin{array}{lllllllll}
0.03 & 0.09 & 0.05 & 0.09 & 0.26 & 0.46 & 0.01 & 0.005
\end{array}\right]^{T}, \\
w_{R} & =\left[\begin{array}{llllllll}
0.13 & 0.50 & 0.08 & 0.10 & 0.05 & 0.06 & 0.04 & 0.04
\end{array}\right]^{T},
\end{aligned}
$$

respectively, are the stable stage distributions of each of the populations. A graphical illustration of the differences between each of these stable stage distributions can be found in Figure 2 in [6]. In [6] the authors note that, while the growth rates for various healthy, diseased and recovered populations are relatively similar, the stable stage distribution for the population predicted from the diseased matrix is much different than that of the healthy and recovered populations, which are quite similar to one another. This was taken to be evidence that recovery was truly occurring due to hypovirus infection. In the following two subsections we describe the tools used to further investigate the differences between these three types of American chestnut populations.

\subsection{Basic reproduction number}

The first line of exploration is to look at population growth and persistence through a different lens. In the traditional way of looking at population persistence for population projection matrix models like (1) one simply compares the growth rate $\lambda$ to 1 : If $\lambda>1$ the population grows without bound, and hence persists indefinitely. If $\lambda<1$ the population decays to zero, and hence goes extinct. If $\lambda=1$ (which is statistically improbable) the population levels off and remains roughly constant as $t \rightarrow \infty$ (and hence persists). For the three populations in [6] the growth rate $\lambda$ is relatively close to one in each case, meaning we can say very little definitively about each population's ability to persist as $t \rightarrow \infty$ using the traditional view, let alone make a comparison about how each population persists in relation to the others.

To view the actual differences in persistence ability between these three populations we need to look at their basic reproduction numbers, which correspond to the average number of offspring per individual per lifetime. To do this we start, like Cushing [4], by decomposing the projection matrix $A$ from (1) into a transition matrix plus a fecundity matrix

$$
A=T+F .
$$

The transition matrix $T$ is composed of the probabilities pertaining to survival and growth of individuals within the population: $i, j$ th entry of the $T$ is the probability of an existing member of the population transitioning from the $j$ th stage to the $i$ th stage in one time-step. Since these transitions cannot generate new individuals for the population, the leading eigenvalue of $T$ is less than one, and hence the matrix $I-T$ is invertible, where $I$ is the 8 by 8 identity matrix. The $i, j$ th entry of the fecundity matrix $F$ is the average number of individuals beginning in stage $i$ created by a member in the $j$ th stage. The basic reproduction number $r$ (or, as Cushing [4] calls it, the inherent net reproductive number) is the leading eigenvalue of the matrix $F(I-T)^{-1}$.

For the models in this paper, Theorem 1.1.3 in [4] states that $r$ is indeed positive, real and unique. Also, since $r$ gives the expected number of offspring per individual per lifetime, it follows that if $\lambda \geq 1$ then $r \geq 1$ and if $\lambda \leq 1$ then $r \leq 1$. Thus, $r$ gives us another 
way to measure whether or not a population is predicted to persist or go extinct. This alternative formulation also gives a clear interpretation of how "far away" a population is from persistence, which makes it preferable in the case where $\lambda<1$ but similar to one. Specifically, while it's not necessarily true that increasing fecundity will be the most efficient or optimal way to increase $\lambda$ (see the Discussion), if $r<1$ simply multiplying the fecundity matrix $F$ by $r^{-1}$ will cause $\lambda=r=1$.

The basic reproduction number $r$ yields a perspective slightly different than the growth rate $\lambda$, due to a more intuitive reference to the lifespan of the average individual within a population. To see this, note that the matrix $(I-T)^{-1}=I+T+T^{2}+\cdots$. One can thus interpret the $i, j$ th entry of $(I-T)^{-1}$ as the expected amount of time than an individual starting in stage $j$ will spend in stage $i$ over the course of its lifetime [4]. Thus, the basic reproduction number $r$, being the 1,1 st element of $F(I-T)^{-1}$ in our case, will be the sum of the average time that an individual starting in stage 1 (first-year juveniles) will spend in each stage multiplied by the average fecundity (number of first-year juveniles produced) by individuals in each stage, summing over all stages. Thus, the basic reproduction number $r$ normalizes population growth/persistence by the average lifespan of individuals within the population. As we will see with the models in [6], this is not a trivial difference, as a population with $\lambda$ near one can have a very small $r$, due in large part to the longevity of the average individual masking deficiencies in its overall ability to at least reproduce itself during its lifetime.

\section{$2.3 \quad$ Population inertia}

The second way we investigate the three types of American chestnut populations is to measure their transient dynamics. To measure transient dynamics stemming from nonasymptotic (equilibrium) stage distributions we use the aforementioned population inertia $p_{\infty}[18,28]$. For a given matrix $A$ and initial population $n_{0}$, population inertia is given by

$$
p_{\infty}=\frac{v^{T} n_{0}}{v^{T} w},
$$

where $v^{T}$ is the leading eigenvector of the transpose of $A, w$ is the aforementioned leading eigenvector (stable stage distribution) of $A$, and $v^{T} w$ is the dot product of $v^{T}$ and $w$. It follows from [2] that $v^{T}$ gives the asymptotic proportion of reproductive output that can be attributed to each size class, so one can view $p_{\infty}$ as the ratio of the actual reproductive output as a result of a population with stage structure $n_{0}$ and the reproductive output of a population at the stable stage distribution. Thus, when $n_{0}=w$, population inertia is one, as expected. A population is said to attenuate when $p_{\infty}<1$ and amplify when $p_{\infty}>1$. The characterizations of attenuation and amplification are less definitive than one would hope, however, given that a population experiencing transient dynamics can oscillate before its growth rate equilibrates. Thus, a population experiencing attenuation may increase at times during its transient phase, but the sum total of decreases outweigh those of the increases, and similar oscillations may occur for a population experiencing amplification.

Notice that, unlike the asymptotic growth rate $\lambda$ or basic reproduction number $r$, population inertia is dependent on the initial population used. Some work has been done to find initial populations $n_{0}$ that give the largest possible transient amplification $\bar{p}_{\infty}$ and attenuation $\underline{p}_{\infty}$, which are especially important when one is not sure of the initial population a priori $[\overline{27}, 28]$. However, we use initial populations that result from different disease transition scenarios. Namely, we will use the $n_{0}=w_{H}$ and $A_{D}$ to study the transient dynamics elicited by going from a healthy population to a diseased (fungus-infected) population and $n_{0}=w_{D}$ and $A_{R}$ to study those elicited by going from diseased (fungus-infected) to recovered (hypovirus-infected). 


\section{Results}

\subsection{Population persistence}

Since the asymptotic growth rates are relatively similar to one another in the healthy, diseased (fungus-infected) and recovered (hypovirus-infected) population models, the focus in Davelos and Jarosz [6] was on the differences in the stable stage distributions (Figure 2 in [6]). The stable stage distribution for the recovered population is very similar to that of the healthy population, both of which are very different than the diseased population, suggesting that recovery from the epidemic has indeed occurred. However, when we take a closer look at the ability of the population to persist in the long run using the basic reproduction number, these differences in persistence ability are not trivial. Both basic reproduction numbers for the diseased $\left(r_{D}=0.57\right)$ and recovered $\left(r_{R}=0.19\right)$ populations are well below one, and the value for the recovered population suggests that the population would need more than five times its current fecundity values to achieve long-term population growth (and hence persistence). This phenomenon is hidden when only looking at $\lambda$ values, as all of these values are near one. One possible explanation for the small discrepency in $\lambda$ values is the relative insensivity of asymptotic population growth rate to fecundity values when the average lifespan of individuals is large, relative to when average lifespans are short [2]. In all three chestnut populations the average lifespan of an individual is on the order of 20-100 years, which is relatively long from an ecological perspective. This long lifespan is normalized away when calculating the basic reproduction numbers, which reveal dire circumstances for the diseased and hypovirus-infected populations, as the average individual cannot come close to reproducing itself during the course of its own lifetime.

\subsection{Transient Dynamics}

So why then are populations with hypovirus infection considered "recovered" if the model predicts that is has a smaller chance of persistence than even a fungus-infected population? The answer is uncovered when looking at transient dynamics. We first look at how the population would respond to changing from being healthy to infected by C. parasitica, by starting with an initial population that is the stable stage distribution for the healthy population, i.e. $n_{0}=w_{H}$, and projecting the population forward using the diseased matrix $A_{D}$. Figures 1(a) and 1(b) and show that, while not all stages see their population sizes decline initially, the total population declines sharply from its initial population size, and eventually each stage's population declines sharply as $t \rightarrow \infty$. Population attenuations are a consequence of the fact that population inertia is 0.73 . These transient attenuations are robust to fecundity values in the healthy population down to roughly 35 percent of their reported values in [6] and all fecundity values for the diseased population less than or equal to those reported (Figure 1(c)). Further, monotonic decline is robust to fecundity values in the healthy population down to roughly 70 percent of their reported values and all fecundity values for the diseased population less than or equal to those reported (see Figure 1(d)). It is important to note that we are assuming that a the population's transition from a healthy state to a diseased state is sudden, as opposed to gradual, which could possibly overexaggerate the short-term transient dynamics elicited by this transition. However, a gradual transition (via incremental perturbations of the projection matrices) will still likely generate population responses that accumulate to the result described above.

Once fecundity values were outside of the aforementioned range of values, population attentuation did not occur. For example, if the fecundity values for the diseased matrix are higher than those reported in [6], there is a possibility of population amplification when transitioning from healthy to diseased. However, fecundity values outside of the aforementioned ranges mimic ecological scenarios that are in the opposite direction or too far away from what is thought to be observed. For example, it is believed that the fecundity values for the diseased matrix in [6] are biased upward, with diseased trees exhibiting a "last-ditch" effort to reproduce before dying, and thus we only considered smaller possible 
fecundity values for the diseased matrix. Additionally, a 30 percent decrease in the fecundity values for the healthy population represents a substantial deviation downward, even with significant uncertainty in the numerical value of these parameters.

On the other hand, if we start with an initial population that is the stable stage distribution for the diseased population, i.e. $n_{0}=w_{D}$, and project the population forward using the recovered matrix $A_{R}$, we see a total population size that amplifies for roughly 25 time-steps before sharply declining as $t \rightarrow \infty$ (Figures 2(a) and 2(b)). Transient amplifications are a consequence of the fact that population inertia is 1.63. These transient amplifications are robust for all fecundity values in the diseased population less than or equal to those reported values in [6], as well as to fecundity values from 40 percent of the recovered population's reported values on up (Figure 2(c)). Large initial amplifications are also robust to fecundity values less than or equal the reported values for the diseased population as well as fecundity values from 70 percent of the recovered population's reported values on up (Figure 2(d)).

Thus, while it appears that recovered populations are indeed recovering in the short term, they have a very high risk for population extinction in the long run. The transient amplification caused by the change in parameter values from a fungus-infected population to a hypovirus-infected population, along with the stage distribution left over from the disease, gives the chestnut population what appears to be a recovery for an extended period of time. In fact, this transient amplification mirrors what would happen if the population were to transition back to a healthy state from a fungus-infected one, as population inertia when using $n_{0}=w_{D}$ as the initial population and $A_{H}$ as the population projection matrix is 1.60 , which is very similar to the 1.63 value above. Thus, in the short term at least, the population dynamics of healthy and hypovirus-infected populations are roughly the same when transitioning from a fungus-infected population, only to diverge as time passes on due to the drastic differences in basic reproduction numbers.

As with the transient attenuation result, fecundity values that are outside of this (relatively wide) range may not produce transient amplification when transitioning from fungusinfected to hypovirus-infected. However, fecundity values for the recovered matrix that are 30 percent smaller than those reported in [6] not only represent a significant deviation downward, but also produce a recovered population that declines asymptotically $(\lambda=0.984$, $r=0.076)$ and does not experience a transient amplification, suggesting that populations with fecundity values less than 70 percent of those reported in [6] are not recovered in any sense.

\section{Discussion}

In this paper we show that short- and long-term measurements of population growth and viability yield a complex picture for American chestnut populations recovering from C. parasitica infection via hypovirus introduction. On one hand, the reappearance of large reproducing chestnut trees, associated with a large proportion of blight-infected isolates being parasitized by the hypovirus, is currently taken to indicate recovery of American chestnut populations [6], leading conservation efforts attempting to introduce hypovirus into existing American chestnut populations throughout the United States. On the other hand, the results of previous work studying the population dynamics of healthy, diseased and recovered American chestnut population are mixed at best, suggesting recovery when examining the stable stage distributions of recovered populations relative to healthy populations, but not necessarily seeing evidence of recovery when studying growth rates [6].

When digging deeper into the dynamics of these populations it becomes clear that recovered populations are recovered only in the short term, with transient dynamics that show large initial population size amplification towards larger values. These transient amplifications are caused by the differences in stable stage structure between diseased and recovered populations. No such amplification is seen when transitioning from healthy to diseased, which was to be expected, given the negative impact blight has been observed to have on 


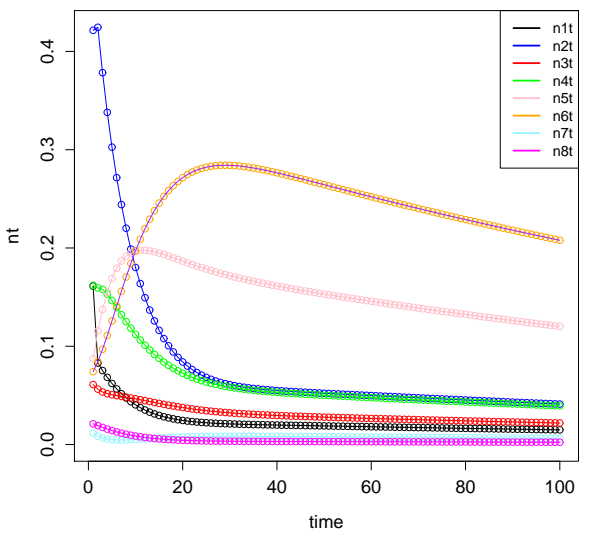

(a)

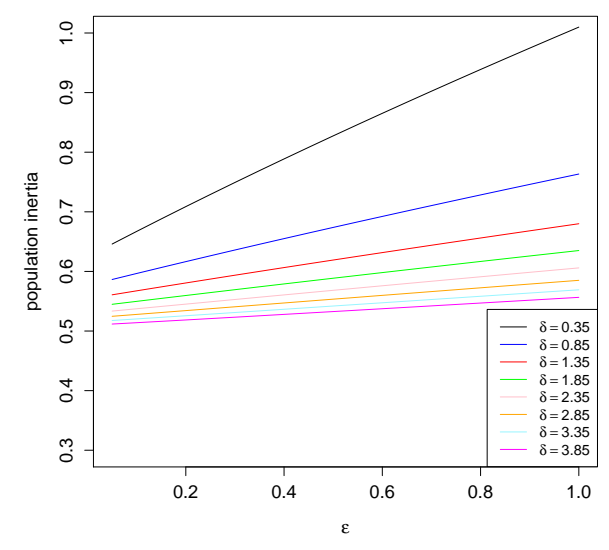

(c)

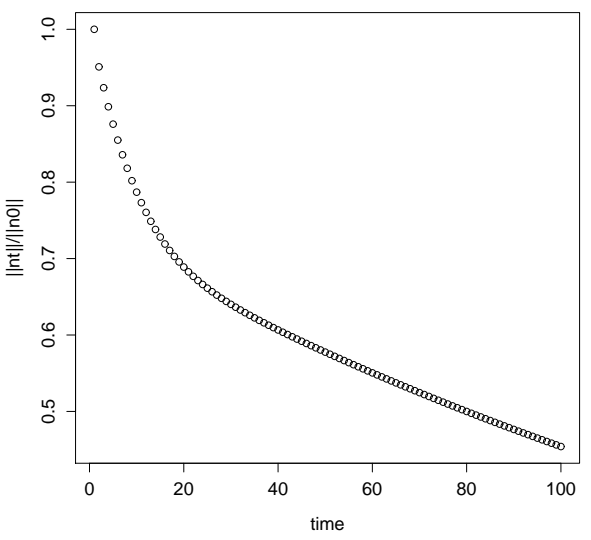

(b)

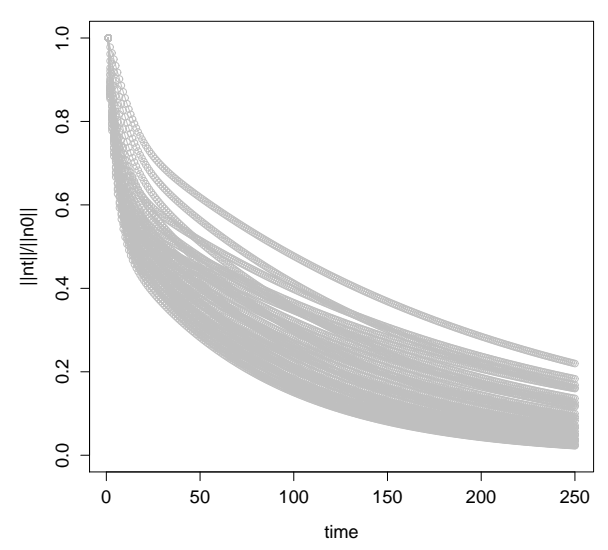

(d)

Figure 1: Population dynamics using $w_{H}$ as the initial population and $A_{D}$ as the population projection matrix. $w_{H}$ is scaled to sum to one so that population numbers can be viewed as a proportion of their initial population. (a) shows the dynamics of each individual stage. (b) gives the dynamics of the total population. (c) and (d) explore of the robustness of the results in (b) to changes in fecundity values in $A_{H}$ and $A_{D}$, using matrices of the form $T_{H}+\delta F_{H}$ and $T_{D}+\epsilon F_{D}$. The terms $\delta$ and $\epsilon$ represent the proportion of the fecundity values used from the matrices $A_{H}$ and $A_{D}$ given in Davelos and Jarosz [6], respectively. (c) gives population inertia values when transitioning from a healthy population to a diseased population as a function of $\epsilon$ for $\delta \in[0.35,3.85]$ in increments of 0.5. (d) Shows population dynamics resulting from using $\epsilon$ values in $[0,1]$ in increments of 0.15 and $\delta$ values in $[0.7,3.5]$ in increments of 0.75 . 


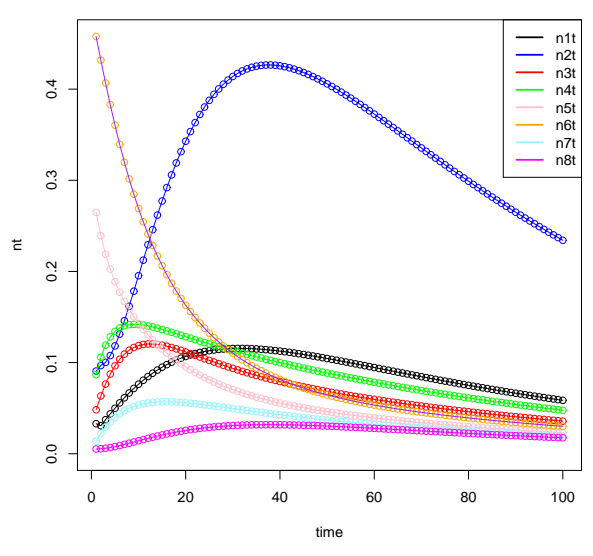

(a)

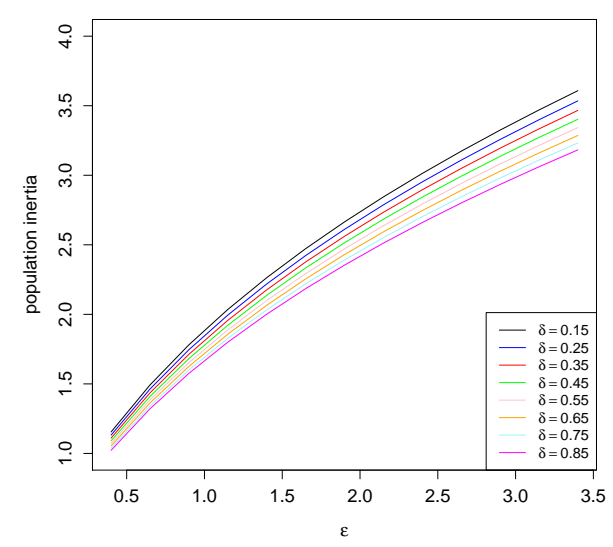

(c)

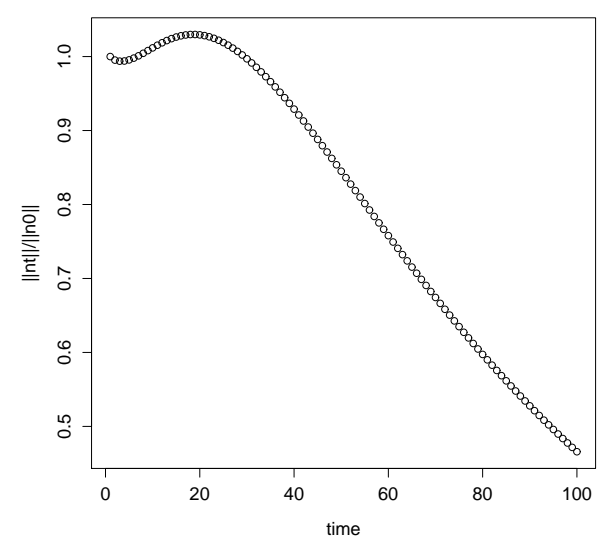

(b)

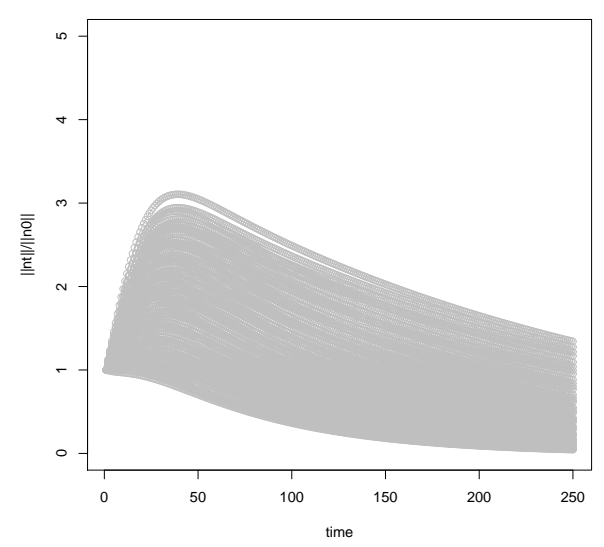

(d)

Figure 2: Population dynamics using $w_{D}$ as the initial population and $A_{R}$ as the population projection matrix. $w_{H}$ is scaled to sum to one so that population numbers can be viewed as a proportion of their initial population. (a) shows the dynamics of each individual stage. (b) gives the dynamics of the total population. (c) and (d) explore of the robustness of the results in (b) to changes in fecundity values, using matrices of the form $T_{D}+\delta F_{D}$ and $T_{R}+$ $\epsilon F_{R}$. (c) gives population inertia values when transitioning from a diseased population to a recovered population as a function of $\epsilon$ for $\delta \in[0.15,0.85]$ in increments of 0.1. (d) Shows population dynamics resulting from using $\epsilon$ values in $[0.75,3.5]$ in increments of 0.25 and $\delta$ values in $[0,1]$ in increments of 0.25 . 
various chestnut communities [22]. Transient amplifications eventually decay away, however, and populations begin to decline in the recovered case, due to both the population growth rate $\lambda_{R}$ and basic reproduction numer $r_{R}$ being smaller than one. This was reported in Davelos and Jarosz [6]. However, because $\lambda_{R}$ was relatively close to one, Davelos and Jarosz [6] did consider the long-term persistence of the recovered populations to be questionable. When viewing persistence from another, and in the long run equivalent, angle using $r_{R}$ one sees that recovered populations are actually quite far off from being persistent, needed more than a five-fold increase in fecundity values to avoid going extinct as $t \rightarrow \infty$.

While this paper mainly focuses on analyzing a population's basic reproduction number, $r$, and population intertia, $p_{\infty}$, values, it is important to note that these analyses were only conducted after the limitations of using the traditional population growth rate, $\lambda$, were met. A thorough study of a population's dynamics should consider (at least) all three of these analysis tools as complimentary means to make the most informed decisions regarding the evaluation of a population's past performances and the predicted results of future conservation measures. Two populations with similar growth rates and basic reproduction numbers may respond drastically differently to the same initial (or current) population, a feature population inertia would be able to detect. On the other hand, two populations with similar population inertias and basic reproduction numbers may have drastically different extinction risks, which traditional population growth rate would be able to detect. For example, of two populations with similar basic reproduction numbers, the shorter-lived population will have a higher population growth rate, and thus a smaller extinction risk if faced with similar transient dynamics. As we have demonstrated in this paper, two populations with similar growth rates can have drastically different basic reproduction numbers if the lifespans of the average individual in both populations is long enough to make the year-to-year discrepancies in population growth appear minimal. Thus, when considering conservation decisions, population biologists should consider the senstivity of all three measurements to different strategies in an attempt to determine the best possible approach to management.

On the positive side, there are multiple, and not mutually exclusive, ways to increase reproduction in American chestnut populations. Most obviously, increasing the number of new seedlings produced per individual would increase the basic reproduction number. Seedling survival and growth can be increased by excluding seed predators and herbivores (e.g. rodents, deer, and turkeys) with cages [5]. The recent explosion in deer population numbers and the cost and time investments in constructing cages make this approach challenging. However, increasing overall reproduction in the American chestnut population need not be entirely focused on fecundity alone. As stated in Section 2.2, while the basic reproduction number provides a definitive reference for how "far away" a population's fecundity is from eliciting population growth, increasing fecundity may not be the most efficient means for increasing this value. Indeed, the basic reproduction number $r_{R}$ for the recovered population is actually most sensitive to changes in stage transitions, specifically the transitions from stage 6 to 7 and from stage 2 to 4 and most elastic to changes in the probability of stage 7 and stage 8 individuals to stay in stages 7 and 8 , respectively (see Figure 3 ). Thus, another strategy would be to change growth dynamics so that trees spend less time in non-reproducing size classes and more time in the reproductive size classes. Changing this growth dynamic could be achieved through a combination of increasing resistance to infection and judicious use of hypovirulence inoculations. A breeding program established by The American Chestnut Foundation has introduced resistance from the Chinese chestnut (Castanea mollisima) into an American chestnut genetic background. These hybrid trees are currently being tested for release into North American forests. Resistance could help trees attain a larger size before becoming infected and then targeted hypovirulence introductions could allow these larger individuals to tolerate the infection and reach reproductive sizes. This combination of resistance and hypovirulence might explain why recovery is widespread and appears to be long lasting in Europe (populations recovering for more than 50 years), since the European chestnuts are slightly less susceptible than American 
chestnuts and hypovirulence has spread naturally and been introduced [16].

Possible extentions of the models in this paper include incorporating density-dependent feedbacks and including demographic and/or environmental stochasticity, which are both research paths currently being taken. Incorporating density-dependent feedbacks will more than likely elicit a model with equilibrium behavior for its population density, as opposed to its growth rate. For the populations in this paper, this would only affect the healthy populations, since the fungus-infected and hypovirus-infected populations are already declining, and density-dependence would not abate this trend toward zero. Some work is currently being done to explore the mathematical properties of nonlinear models with density-dependent feedbacks impacting fecundity [8], which draws on the previous work of $[23,31,10,25]$. Stochastic models analogous to those in this paper will have long-term average growth rates $\lambda_{s}$, the properties of which have been studied extensively in the theoretical ecology literature (see, for example, $[2,32]$ ). However, the transient dynamics of stochastic models are far less understood (but see [13]), and extending the work in this paper to stochastic models for American chestnuts is in progress.

The results of our study illustrate the importance of taking a long-term view of recovery for American chestnuts. While current approaches have provided a transient amplification and are necessary for the recovery of populations, to maintain the population level gains we have made in the long run we need to broaden our emphasis to include increasing reproduction of individuals and increasing the time individuals spend in reproductive classes and the successful recruitment of seedlings.

$$
\begin{aligned}
S & =\left(\begin{array}{cccccccc}
0 & 0 & 0 & 0 & 0 & 0.20 & 0.07 & 0.05 \\
0.27 & 1.00 & 0 & 0 & 0 & 0 & 0 & 0 \\
0 & 0 & 0.37 & 0.55 & 0.21 & 0.20 & 0 & 0 \\
0 & 9.48 & 0.94 & 1.43 & 0.53 & 0.51 & 0.19 & 0 \\
0 & 0 & 0 & 4.31 & 1.61 & 1.54 & 0.57 & 0 \\
0 & 0 & 0 & 0 & 3.30 & 3.17 & 1.17 & 0.83 \\
0 & 0 & 0 & 0 & 0 & 12.0 & 4.43 & 3.16 \\
0 & 0 & 0 & 0 & 0 & 0 & 7.27 & 5.20
\end{array}\right) \\
E & =\left(\begin{array}{ccccccccc}
0 & 0 & 0 & 0 & 0 & 0.02 & 0.30 & 0.68 \\
1.00 & 4.26 & 0 & 0 & 0 & 0 & 0 & 0 \\
0 & 0 & 1.58 & 0.29 & 0.04 & 0.01 & 0 & 0 \\
0 & 1 & 0.35 & 5.87 & 0.25 & 0.05 & 0.00 & 0 \\
0 & 0 & 0 & 1.36 & 6.61 & 0.49 & 0.01 & 0 \\
0 & 0 & 0 & 0 & 1.57 & 14.9 & 0.25 & 0.02 \\
0 & 0 & 0 & 0 & 0 & 1.27 & 21.3 & 0.83 \\
0 & 0 & 0 & 0 & 0 & 0 & 1.53 & 25.87
\end{array}\right)
\end{aligned}
$$

Figure 3: The sensitivity and elasticity of the basic reproduction number $r_{R}$ for the recovered population. The $i, j$ th element of $S$ is the sensitivity of $r_{R}$ to changes in the $i, j$ th element of $A_{R}$, and $i, j$ th element of $E$ is the elasticity of $r_{R}$ to changes in the $i, j$ th element of $A_{R}$.

\section{References}

[1] M. G. Buhnerkempe, N. Burch, S. Hamilton, K. M. Byrne, E. Childers, K. A. Holfelder, L. N. McManuse, M. I. Pyne, G. Schroeder, and P. F. Doherty Jr. The utility of transient senstivity for wildlife management and conservation: bison as a case study. Biological Conservation, 144: 1808-1815, 2011.

[2] H. Caswell, Matrix population models : construction, analysis, and interpretation. Sinauer Associates, 2001. 
[3] H. Caswell. Sensitivity analysis of transient population dynamics. Ecology Letters, 10(1): 1-15, 2007.

[4] J. M. Cushing. An introduction to structured population dynamics. Society for Industrial and Applied Mathematics, 1998.

[5] A. L. Davelos. Double-stranded RNA mediated recovery of American chestnut populations: a demographic analysis. $\mathrm{PhD}$ thesis, Michigan State University, East Lansing, Michigan, 1999.

[6] A. L. Davelos and A. M. Jarosz. Demography of American chestnut populations: effects of a pathogen and a hyperparasite. Journal of Ecology, 92: 675-685, 2004.

[7] A. Davelos Baines, D. W. Fulbright, and A. M. Jarosz. Effects of branch size and pathogen virulence on canker development and branch mortality. Acta Horticulturae, 1019:23-29, 2014.

[8] E. A. Eager and R. Rebarber. Modeling and mathematical analysis of populations with adult-conspecific density-dependence. In preparation.

[9] E. A. Eager, R. Rebarber, and B. Tenhumberg. Choice of density-dependent seedling recruitment function affects predicted transient dynamics: a case study with Platte thistle. Theoretical Ecology, 5(3): 387-401, 2012.

[10] E. A. Eager, R. Rebarber, and B. Tenhumberg. Global asymptotic stability of plant-seed bank models. Journal of Mathematical Biology, 69: 1-37, 2014.

[11] T. H. G. Ezard, J. M. Bullock, H. J. Dalgleish, A. Millon, F. Pelletier, A. Ozgull, and D. N. Koons. Matrix models for a changeable world: the importance of transient dynamics in population management. Journal of Applied Ecology, 47(3):515-523, 2010.

[12] D. W. Fulbright, W. H. Weidlich, K. Z. Haufler, C. S. Thomas, and C. P. Paul. Chestnut blight and recovering American chestnut trees in Michigan. Canadian Journal of Botany, 61:3164-3171, 1983.

[13] C. V. Haridas and S. Tuljapurkar. Time, transients and elasticity. Ecology Letters 10: 1143-1153, 2007.

[14] A. Hastings. Transients: the key to long-term ecological understanding? Trends in Ecology and Evolution, 19(1): 39-45, 2004.

[15] D. He, Q-G. Wang, S. B. Franklin, and M-X. Jiang. Transient and aysmptotic demographics of the riparian species. Euptelea pleiospermum in the Shennogjia area, central China. Biological Conservation, 161: 193-202, 2013.

[16] U. Heiniger and D. Rigling. Biological control of chestnut blight in Europe. Annual Review of Phytopathology, 32:581599, 1994.

[17] D. N. Koons, J. B. Grand, J. B. Zinner, and B. Rockwell. Transient population dynamics: relations to life history and initial population state. Ecological Modelling, 185: 283-297, 2005.

[18] D. N. Koons, R. R. Holmes, and J. B. Grand. Population inertia and its sensitivity to changes in vital rates and population structure. Ecology, 88(11): 2857-2867, 2007.

[19] W. L. MacDonald and D. W. Fulbright. Biological control of chestnut blight: Use and limitations of transmissible hypovirulence. Plant Disease,75:656-661, 1991.

[20] S. M. McMahon and C. J.E. Metcalf. Transient sensitivities of non-indigenous shrub species indicate complicated invasion dynamics. Biological Invasions, 10(6): 833-846, 2008. 
[21] H. W. Merkel. A deadly fungus on the American chestnut. 10th Annual Report of the New York Zoological Society, Bronx. pp. 97-103, 1905.

[22] M. G. Milgroom and P. Cortesi. Biological control of chestnut blight with hypovirulence: A Critical Analysis. Annual Review of Phytopathology,42:311-338, 2004.

[23] R. Rebarber, B. Tenhumberg, and S. Townley. Global asymptotic stability of density dependent population projection models. Theoretical Population Biology 81 81-87, 2012.

[24] J. R. Saucier. American chestnutan American wood. USDA Forest Service FS. 230, 1973.

[25] H. L. Smith and H. R. Thieme. Persistence and global stability for a class of discrete time structured population models. Discrete and Continous Dynamtical Systems Series $A$ (DCDS - A), 2013

[26] I. Stott, M. Franco, D. Carslake, S. Townley, and D. Hodgson. Boom or bust? A comparative analysis of transient population dynamics in plants. Journal of Ecology, 92(2): 302-311, 2010.

[27] I. Stott, S. Townley, and D. Hodgson. A framework for studying transient dynamics of population projection matrix models. Ecology Letters, 14: 959-970, 2011.

[28] I. Stott, D. J. Hodgson, and S. Townley. Beyond sensitivity: nonlinear perturbation analysis of transient dynamics. Methods in Ecology and Evolution, 3: 673-684, 2012.

[29] S. Townley, D. Carslake, O. Kellie-Smith, D. McCarthy, and D. Hodgson. Predicting transient amplification in perturbed ecological systems. Journal of Applied Ecology: 45(6): 1243-1251, 2007.

[30] S. Townley and D. Hodgson. Errtum et addendum: transient amplification and attenuation in stage-structured population dynamics. Journal of Applied Ecology: 45(6): 1836-1839, 2008.

[31] S. Townley, B. Tenhumberg, R. Rebarber. Feedback control systems analysis of density dependent population dynamics. Systems and Control Letters Syst 61 309-315, 2012

[32] S. Tuljapurkar, C. C. Horvitz, and J. B. Pascarella. The many growth rates and elasticities of populations in random environments. The American Naturalist 162(4): 489-502, 2003. 